\title{
https://tecnohumanismo.online
}

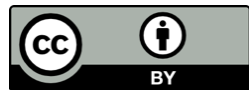

\section{Actividad hidrolítica de la amilasa sanguínea en pacientes altoandinos aparentemente sanos - Ayacucho, Perú}

Hydrolytic activity of blood amylase in apparently healthy high Andean patients - Ayacucho, Peru Atividade hidrolítica da amilase sanguínea em pacientes aparentemente saudáveis do Alto Andino - Ayacucho, Peru

\section{ARTÍCULO GENERAL}

Jesús Javier Ñaccha Urbano

jesus.naccha@unsch.edu.pe

https://orcid.org/0000-0002-5035-4849

Universidad Nacional de San Cristóbal de Huamanga,

Ayacucho - Perú
Magdalena Cunto Arone
magdalenaca2@hotmail.com

https://orcid.org/0000-0002-1931-7847

Universidad Nacional de San Cristóbal de Huamanga, Ayacucho - Perú

Recibido 15 de Febrero 2021 | Arbitrado y aceptado 03 de Marzo 2021 | Publicado el 01 de Junio 2021

\section{RESUMEN}

El objetivo general de esta investigación es determinar la actividad hidrolítica de la amilasa sanguínea en pacientes altoandinos aparentemente sanos, de Ayacucho, Perú. La metodología utilizada se basó en un diseño no experimental, de campo, con un nivel descriptivo y explicativo. Como conclusión se puede indicar que, de los 70 pacientes estudiados, el promedio de edad y peso su valor de amilasa en las 35 mujeres fue de 125 $\mathrm{UA} / \mathrm{dl}, 26$ años de edad y $58 \mathrm{Kg}$ de peso, y en los 35 varones de $142 \mathrm{UA} / \mathrm{dl}$ con 28 años y peso de $64 \mathrm{Kg}$, notando que los jóvenes del sexo masculino son superiores a los valores normales de $120 \mathrm{UA} / \mathrm{dl}$. Referente a la edad se observa que los de 17 a 23 años hay más mujeres $17(24.3 \%)$ con valores de amilasa de $97.9 \mathrm{UA} / \mathrm{dl}$, donde hay más varones 13 (18.6\%) entre 24 a 30 años con $110.5 \mathrm{UA} / \mathrm{dl}$. El sector de mayor de edad es de 31 a 36 años solamente hay 7 mujeres (11\%) con $146.4 \mathrm{UA} / \mathrm{dl}$ y 11 varones $(15.7 \%) 150.6$ $\mathrm{UA} / \mathrm{dl}$, ambos casos superiores, por consumo de alimentos chatarra y alcohol de ambos sexos, son proclives a contraer la pancreatitis. Con relación al índice de masa corporal, se muestra que en su mayoría de los jóvenes presentan un normopeso: 18 pacientes $(25.7 \%)$ de mujeres $120.6 \mathrm{UA} / \mathrm{dl}$, pero estadísticamente no significativa. Respecto a los 14 varones $(20 \%)$ con actividad amilásica 177.7 UA/dl presenta una pequeña variación de la actividad enzimática, superior a los valores normales.

Palabras clave: amilasa, enzima, edad, peso, pancreatitis.

\section{ABSTRACT}

The general objective of this research is to determine the hydrolytic activity of blood amylase in apparently healthy high Andean patients from Ayacucho, Peru. The methodology used was based on a nonexperimental, field design, with a descriptive and explanatory level. As a conclusion, it can be indicated that, of the 70 patients studied, the average age and weight, their amylase value in the 35 women was $125 \mathrm{AU} / \mathrm{dl}, 26$ years of age and $58 \mathrm{~kg}$ of weight, and in the 35 men of $142 \mathrm{AU} / \mathrm{dl}$ at 28 years of age and weighing $64 \mathrm{~kg}$, noting that young males are higher than the normal values of $120 \mathrm{AU} / \mathrm{dl}$. Regarding age, it can be seen that those between 17 and 23 years old have more women $17(24.3 \%)$ with amylase values of $97.9 \mathrm{AU} / \mathrm{dl}$, where there are more men 13 (18.6\%) between 24 and 30 years with 110.5 $\mathrm{AU} / \mathrm{dl}$. The older sector is from 31 to 36 years old, there are only 7 women $(11 \%)$ with $146.4 \mathrm{AU} / \mathrm{dl}$ and 11 men (15.7\%) 150.6 $\mathrm{AU} / \mathrm{dl}$, both higher cases, due to consumption of junk food and alcohol of both sexes, are prone to pancreatitis. Regarding the body mass index, it is shown that most of the young people have a normal weight: 18 patients $(25.7 \%)$ of women 120.6 $\mathrm{AU} / \mathrm{dl}$, but not statistically significant. Regarding the 14 males (20\%) with amylase activity $177.7 \mathrm{AU} / \mathrm{dl}$, there is a small variation in enzymatic activity, higher than normal values.

Keywords: amylase, enzyme, age, weight, pancreatitis.

\section{RESUMO}

O objetivo geral desta pesquisa é determinar a atividade hidrolítica da amilase sangüínea em pacientes altos andinos aparentemente saudáveis de Ayacucho, Peru. A metodologia utilizada assentou num desenho de campo não experimental, com um nível descritivo e explicativo. Como conclusão, pode-se indicar que, dos 70 pacientes estudados, a média de idade e peso, seu valor de amilase nas 35 mulheres foi de $125 \mathrm{UA} / \mathrm{dl}, 26$ anos de idade e $58 \mathrm{~kg}$ de peso, e nos 35 homens de $142 \mathrm{UA} / \mathrm{dl}$ aos 28 anos de idade e pesando $64 \mathrm{~kg}$, observando que os jovens do sexo masculino estão acima dos valores normais de 120 UA/dl. Em relação à idade, pode-se observar que aqueles entre 17 e 23 anos têm mais mulheres $17(24,3 \%)$ com valores de amilase de 97,9 UA/dl, onde há mais homens $13(18,6 \%)$ entre 24 e 30 anos com $110,5 \mathrm{UA} / \mathrm{dl}$. O setor mais velho é de 31 a 36 anos, são apenas 7 mulheres (11\%) com 146,4 UA/dl e 11 homens $(15,7 \%)$ 150,6 UA/dl, ambos casos mais elevados, devido ao consumo de junk food e álcool de ambos os sexos, são propensos a pancreatite. Em relação ao índice de massa corporal, mostra-se que a maioria dos jovens tem peso normal: 18 pacientes $(25,7 \%)$ das mulheres $120,6 \mathrm{UA} / \mathrm{dl}$, mas sem significância estatística. Em relação aos 14 homens (20\%) com atividade amilase 177,7 UA/dl, há uma pequena variação na atividade enzimática, acima dos valores normais.

Palavras-chave: amilase, enzima, idade, peso, pancreatite. 


\section{Introducción}

La amilasa es una enzima producida por el organismo para digerir los alimentos, esta es generada en mayor cantidad en el páncreas y en las glándulas salivales, y puede estar presente en menor proporción en la orina, trompas de falopio, ovario, próstata, y el intestino, "las amilasas de origen pancreático y salival se abrevian como (isoenzima, isoamilasa) tipo P y tipo S" (Armijo Zambrano \& Atupaña Bacuy, 2018, pág. 7), ambas están estrechamente relacionadas pero con diferencias órgano específicas, proporcionando mapas peptídicos similares mas no idénticos, el peso molecular de la amilasa pancreática es de 54.000 y la salival tiene un peso mayor.

Las amilasas son metaloenzimas que contiene al menos un átomo de calcio por molécula; este metal es importante ya que actúa en su actividad metabólica. El pH de la actividad óptima se encuentra entre 6,9 y 7 . El pH óptimo para la amilasa salival depende del anión que se emplee como activador, siendo el más importante el cloruro. (Armijo Zambrano \& Atupaña Bacuy, 2018, pág. 8)

Esta enzima es secretada por el páncreas a través de sus células acinares, que luego de pasar por el conducto pancreático se dirige al duodeno y de allí al intestino, (Armijo Zambrano \& Atupaña Bacuy, 2018), de manera que cuando la amilasa llega a esta parte del organismo es cuando los hidratos de carbono son transformados en azucares simples por medio de la digestión, labora que realiza la alfa amilasa por medio de la actividad hidrolítica, de igual manera la amilasa es excretada por la orina a través del sistema renal.

Como lo indica Garboa Pérez \& Ochoa Díaz, (2017), la amilasa, son enzimas glucolíticas que hidrolizan los enlaces éter (glucósidicos) de las cadenas de polisacáridos de las sustancias amiláceas, degradándolas a oligosacáridos polisacáridos $\mathrm{y}$ monosacáridos que son más solubles en medio acuosos. En la Tabla 1 se describe la clasificación de la amilasa.

Tabla 1

Clasificación de la Amilasa

\begin{tabular}{ll}
\hline CLASIFICACION & CARACTERISTICA \\
\hline \multirow{3}{*}{ Alfa amilasa } & Cataliza la hidrolisis al azar los enlaces $\alpha$ \\
& $\begin{array}{l}1,4 \text { glucosídicos de la región central de la } \\
\text { cadena de amilosa y amilopectina }\end{array}$ \\
\hline
\end{tabular}


exceptuando las moléculas cercanas a la ramificación obteniendo como resultados maltosa y oligosacáridos de varios tamaños.

Es una exoenzima que ataca a los enlaces $\alpha 1,4$ glucosídicos en la parte externa de la cadena del almidón, la $\beta$ amilasa separa

Beta Amilasa unidades de maltosa a partir de los exámenes no reductores de esta por hidrolisis alterna de los enlaces glucosídicos.

Fuente: (Garboa Pérez \& Ochoa Díaz, 2017)

Los numerosos métodos descritos para la determinación de la $\alpha$-amilasa miden la reducción del sustrato de forma viscosimétrica, turbidimétrica nefelométrica $\mathrm{o}$ amiloclástica o bien registran la formación de productos de degradación por sacarimetría o cinéticamente por reacciones sucesivas catalizadas por enzimas...la $\beta$-amilasa es también sintetizada por bacterias, hongos y plantas. Los tejidos animales no contienen $\beta$ amilasa, aunque puede estar presente en microorganismos saprófitos del tracto gastrointestinal. Tiene un $\mathrm{pH}$ óptimo de 12. El procedimiento a nivel laboratorio es utilizar tres métodos distintos: - "salting out" con sulfato de amonio - cromatografía en columna de DEAE-celulosa - filtración en gel utilizando Sephadex G-200. E1 uso más importante es la producción de jarabes y en la industria de la panificación, ya que hidrolizan el almidón y producen los azúcares (glucosa y maltosa). (Garboa Pérez \& Ochoa Díaz, 2017, pág. 28)

Cuando la amilasa se encuentra en gran cantidad o de manera insuficiente en el individuo puede indicar alguna alteración en el páncreas, alguna infección, alcoholismo, o cualquier otro problema médico, (Tabernilla, Tabernilla, Guerra, \& Díaz, 2019). Dichos valores se obtienen a través de pruebas de laboratorio en los cuales si arrojan un nivel bajo de amilasa este podría indicar que el paciente tiene una pancreatitis aguda, una inflamación en el páncreas, que puede provocar daños irreversibles con el tiempo, todo esto puede ser generado por el consumo excesivo de alcohol, enfermedad hepática y fibrosis quística. 
De la misma manera si los valores de amilasa se encuentran elevados en el organismo es indicativo de afecciones como obstrucción en el páncreas, presencia de cáncer pancreático, o una inflamación en el páncreas que si es tratada con rapidez puede mejorar en poco tiempo.

Una amilasa total con valores 10 veces o más por encima del límite superior de referencia es sugestiva de una pancreatitis. De cinco a diez veces el valor normal puede indicar una afección del íleo o duodeno, o un fallo renal, y elevaciones más bajas son hallazgos frecuentes en enfermedades de las glándulas salivales. (Tabernilla, et al., 2019, pág. 76)

Así mismo, Tabernilla et al., (2019) indica que se han encontrado algunos casos de macroamilasa, que es cuando la amilasa se une a otra molécula, lo que provoca una disminución de la cantidad excretada por la orina, generando concentraciones mayores en sangre, sin encontrar una causa aparente, como también en pacientes con enfermedad renal, causando que los niveles de concentración de la amilasa sean elevados en su organismo aun cuando su eliminación es normal por sus riñones.

A continuación, se demostrarán algunas causas que generan la hiperamilasemia (ver tabla 2)

\section{Tabla 2}

Causas de la Hiperamilasemia

\begin{tabular}{ll}
\hline ENFERMEDADES DEL PÁNCREAS & $\begin{array}{l}\text { SÍNDROMES ABDOMINALES } \\
\text { AGUDOS EXTRAPANCREÁTICOS }\end{array}$ \\
\hline -Pancreatitis aguda biliar & -Perforación de úlcera \\
-Pancreatitis aguda alcohólica sobre & -Colecistitis \\
pancreatitis crónica & -Obstrucción intestinal, Infarto de \\
-Colangiopancreatografía retrógrada & mesenterio \\
endoscópica (CPRE) & -Apendicitis aguda \\
-Pancreatitis aguda farmacológica & -Peritonitis de diversas causas \\
-Traumatismo & -Embarazo extrauterino \\
-Cáncer pancreático & -Salpingitis \\
-Obstrucción ductal & -Disección aórtica \\
-Complicaciones de pancreatitis & -Insuficiencia renal \\
(pseudoquistes, abscesos) & -Gastroenteritis severa \\
\hline APARTE DE UN SÍNDROME & -Enfermedad celiaca \\
ABDOMINAL AFECCIONES DE LAS & FÁRMACOS \\
\hline
\end{tabular}


GLÁNDULAS SALIVALES Y PAROTÍDEAS EVIDENTE

-Parotiditis

-Litiasis salival

-Estimulación de las glándulas salivales y parotídeas por: alcoholismo, bulimia, posfibroendoscopia gástrica.

Producción ectópica por tumores:

próstata, ovarios, pulmón, esófago, mama y timo.

Insuficiencia renal crónica

Macroamilasemia

Acidosis (cetónica y no cetónica)

\section{Aumentan}

-hormona adrenocorticotropica, ácido amino salicílico, algunos antibióticos (ej. nitrofurantoína), antineoplásicos (ej. asparginasa), aspirina, atovaquone, sales de calcio, sales de cloruro, clorpromazina, chlorthalidone, colinérgicos (ej. betanecol), cimetidina, codeína, ciproheptadina, didanosina, estrógenos, etanol, ácido etacrínico, fluoruros, contraste que contienen iodo, metildopa, metoclopramida, ranitidina, sulfonamidas, diuréticos tiazídicos, ácido valproico, etc.

Disminuyen

-citratos, dextrosa intravenosa, oxalatos, saquinavir

Fuente: (Orlando, 2016)

Los hidratos de carbono conformar un mayor porcentaje en la alimentación a nivel mundial, aportando más del $50 \%$ de calorías en la nutrición diaria, y estos a su vez requieren de un proceso de digestión más fuerte y complejo; para el caso de la población altoandina, el consumo de carbohidratos conforma en gran parte su dieta diaria, (Huaman Apaza \& Rodríguez Huaman, 2018), los cuales a su vez se convierten en fuente de energía para poder realizar las actividades relacionadas con la agricultura que ejecutan los pobladores de estas regiones.

Todos los mamíferos tienen la capacidad para convertir los carbohidratos como la sacarosa, el almidón, la lactosa y maltosa en glucosa de esa manera obtener energía o almacenarlas como glucógeno; a su vez la glucosa puede convertirse en colesterol, en grasas incluso en proteínas. Tanto los hidratos de carbono y las grasas nos proporcionan energía para mantener la temperatura corporal y para diversos procesos internos por lo tanto estos nutrientes, al igual que el agua, se necesita en mayor cantidad que los otros. Los hidratos de carbono son provistos por el azúcar, los cereales, tubérculos, raíces y algunas frutas. Las principales fuentes de grasa son los aceites, mantequillas, margarina y manteca. (Huaman Apaza \& Rodríguez Huaman, 2018) 
Muchos seres vivos y las plantas tienen como forma de reserva al almidón. A diferencia de la celulosa que le confiere la dureza al reino vegetal ya que la pared celular está conformada por ella, (Restrepo \& Tascon, 2013, pág. 2), por tanto los humanos, y la mayoría de los animales, no son capaces de eliminar los enlaces beta de la celulosa, y por eso se les denomina "fibra" y al no ser absorbidos por los intestinos, lo que hace es acelerar el tránsito intestinal, a diferencia de los enlaces alfa del almidón que son cortados sin complicación.

Para analizar los valores de amilasa en sangre se utilizan diversos métodos entre ellos la espectrometría, que por medio del análisis químico y el uso de un equipo determinado espectrómetro emite un haz de luz monocromático sobre la muestra (Basantes Procel, 2014), así se mide la cantidad de luz que es absorbida por la muestra y le permite al evaluador determinar la cantidad de la sustancia procesada en este caso la amilasa y poder determinar los valores arrojados.

Los valores referenciales en sangre de la amilasa, normalmente están situados entre 25 a 115U/L, aunque estos varían de acuerdo a las diversas casas comerciales que suministran los reactivos y a su vez estos están establecidos de acuerdo al país, tipo de población, raza y costumbre (Armijo Zambrano \& Atupaña Bacuy, 2018), y es que mediante la determinación de la amilasa sérica se pueden diagnosticar diversas patologías en base a los resultados obtenidos.

Para finalizar se indica que el objetivo general planteado en esta investigación es determinar la actividad hidrolítica de la amilasa sanguínea en pacientes altoandinos aparentemente sanos en Ayacucho, Perú. La metodología utilizada se basó en un diseño no experimental, de campo, con un nivel descriptivo y explicativo.

\section{Metodología}

La metodología aplicada para esta investigación es de diseño no experimental. El cuál es el que se realiza sin manipular en forma deliberada ninguna variable, donde se observan los hechos tal como se presentan en su contexto real y en un tiempo determinado o no, para luego analizarlos (Palella Stracuzzi \& Martins Pestana, 2010). Por lo que en 
este diseño no se construye una situación específica, sino que se observan las que ya existen.

Los objetivos de esta investigación se basaron en conocer la actividad de la amilasa sanguínea en pacientes aparentemente sanos, establecer diferencias comparativas y la significancia correspondiente entre los valores hallados en el laboratorio, evaluar y diagnosticar los factores de la actividad enzimática en función a la edad, peso sexo, procedencia, y cuantificar la presencia de amilasa sérica.

El tipo de investigación a realizar es de campo. Esta consiste en la recolección de datos directamente de la realidad donde ocurren los hechos, sin manipular o controlar variables, así como también permite indagar los efectos de la interrelación entre los diferentes tipos de variables en el lugar de los hechos (Palella Stracuzzi \& Martins Pestana, 2010).

De la misma manera el nivel de la investigación es descriptivo, cuyo propósito es el de interpretar realidades de hecho. Incluye descripción, registro, análisis e interpretación de la naturaleza actual, composición o procesos de los fenómenos (Palella Stracuzzi \& Martins Pestana, 2010).

Así mismo, otro nivel de investigación es el explicativo. Este se centra en determinar los orígenes o las causas de un determinado conjunto de fenómenos complejos y delicados, cuyo objetivo es el de encontrar relaciones causa-efecto de ciertos hechos con el objeto de conocerlos con mayor profundidad.

\section{Población y Muestra.}

La medida de la población y muestra que será estudiada, es una parte importante en el desarrollo de una investigación. La población es el conjunto de unidades de las que se desea obtener información y sobre las que se van a generar conclusiones, en otra forma, es el conjunto finito o infinito de elementos, personas o cosas pertenecientes a la investigación (Palella Stracuzzi \& Martins Pestana, 2010).

La población de esta investigación está constituida por los habitantes altoandinos de Ayacucho, Perú. La muestra tomada es de 70 pacientes divididos en 35 mujeres y 35 hombres, estructurados para su análisis según la edad, sexo, peso y procedencia. 


\section{Resultados y discusión}

En la Tabla 3 se pude observar el promedio general de la actividad amilásica sérica $\mathrm{UA} / \mathrm{dl}$ en pacientes altoandinos aparentemente sanos en ambos sexos.

\section{Tabla 3}

Promedio general de la actividad amilásica sérica UA/dl en pacientes altoandinos aparentemente sanos en ambos sexos.

\begin{tabular}{lll}
\hline PROMEDIOS & $\begin{array}{l}\text { SEXO } \\
\text { FEMENINO }\end{array}$ & $\begin{array}{l}\text { SEXO } \\
\text { MASCULINO }\end{array}$ \\
\hline Amilasa sérica & $125 . \mathrm{UA} / \mathrm{dl}$ & $142 . \mathrm{UA} / \mathrm{dl}$ \\
\hline Edad & 26 años & 28 años \\
\hline Peso & $58 \mathrm{~kg}$ & $64 \mathrm{~kg}$ \\
\hline
\end{tabular}

Es notorio el promedio general obtenido de la actividad amilásica sérica en US/100 $\mathrm{ml}$ en pacientes altoandinos aparentemente sanos en la tabla 3. Siendo el sexo femenino con $125 \mathrm{UA} / \mathrm{dl}$, de 26 años y $58 \mathrm{~kg}$ promedio. Hay un ligero incremento en el peso debido a la ingestión de alimentos a base de carbohidratos especialmente la comida chatarra. Siendo superior los promedios del sexo masculino con $14 \mathrm{UA} / \mathrm{dl}, 28$ años y $64 \mathrm{~kg}$ de peso, lo que significa que posiblemente se alimentan con carbohidratos presentes en la comida al paso, pero si son bohemios consumidores de las bebidas alcohólicas.

Del mismo modo, la variación de la actividad amilásica en UA/dl pacientes altoandinos aparentemente sanos según los grupos etarios, se distingue en la Figura 1.

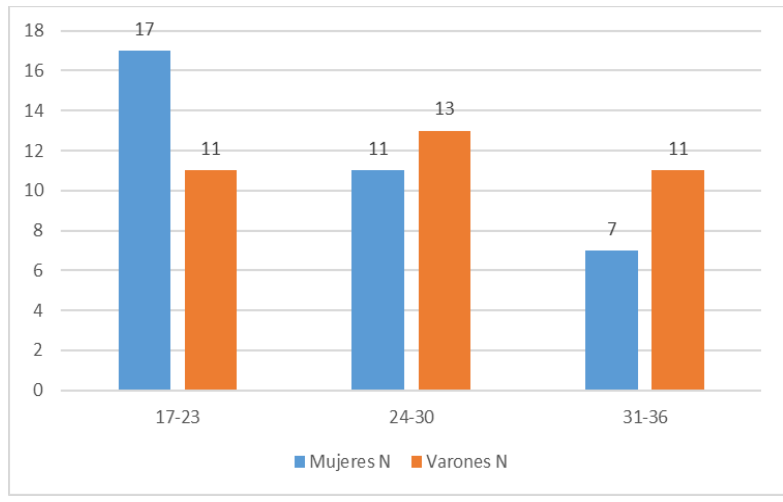

Figura 1. Variación de la actividad amilásica en UA/dl pacientes altoandinos aparentemente sanos según los grupos etarios. 
En consideración al grado de amilasa de pacientes altoandinos aparentemente sanos en relación al grupo etario visto en la figura 1, se deduce que la mayor cantidad de mujeres se encuentran en el grupo etario 17 a 23 años con 17 individuos (24.5\%). En tanto los varones se ubican en gran proporción en el grupo etario de 24 a 30 años con 13 individuos $(18.6 \%)$ lo que significa que los varones tienen responsabilidades o familia y trabajan a medio tiempo para cubrir sus necesidades de ahí se interpreta la mayoría de edad que las mujeres.

También encontramos en menor cantidad en el grupo de 31 a 36 años para ambos sexos, que son personas ya tienen una familia o son padres y madres solteras que hacen los esfuerzos posibles por concluir sus estudios.

En el mismo sentido, en la Figura 2 se puede visualizar la variación de actividad amilásica en pacientes altoandinos aparentemente sanos según el índice de masa corporal.

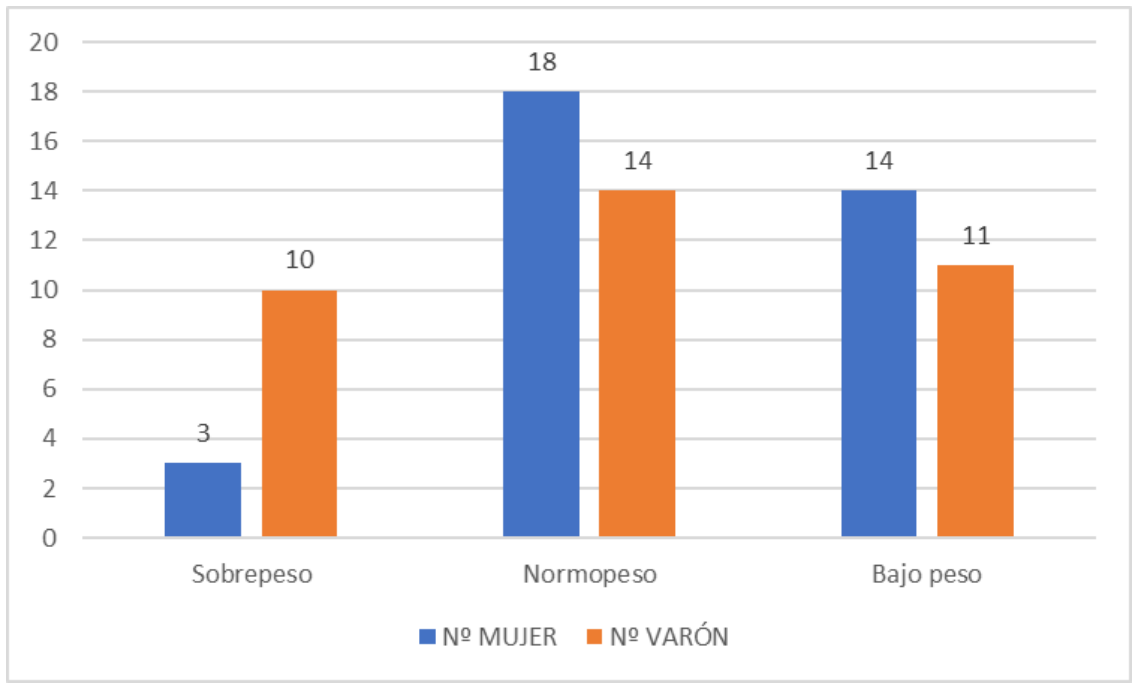

Figura 2. Variación de la actividad amilásica en pacientes altoandinos aparentemente sanos según el índice de masa corporal.

La determinación de la amilasa sérica en pacientes altoandinos aparentemente sanos en ambos sexos en función al índice de masa corporal se muestra en la figura 2. Podemos observar que personas con sobrepeso son, 3 mujeres (4.3\%) y los 10 varones (14.3\%) lo que indica que esta cantidad es superior a las mujeres consideramos que el sedentarismo y el consumo de comida chatarra y las bebidas alcohólicas lo permiten. Con peso normal o normopeso se encuentra 18 mujeres (25.7\%) y 14 varones (20\%) son jóvenes en actividad física constante. Es preocupante notar personas con bajo peso tal es el caso de 
14 mujeres (20\%) y 11 varones (15.7\%) la situación económica que presentan los jóvenes no permite alimentarse bien, cabe mencionar que muchos estudiantes universitarios de bajos recursos por la comida, otros debido a sus quehaceres y deberes se olvidan de alimentarse en su debido momento.

Así mismo, la variación de la actividad amilásica en pacientes altoandinos aparentemente sanos en relación a la procedencia se muestra en la Figura 3.

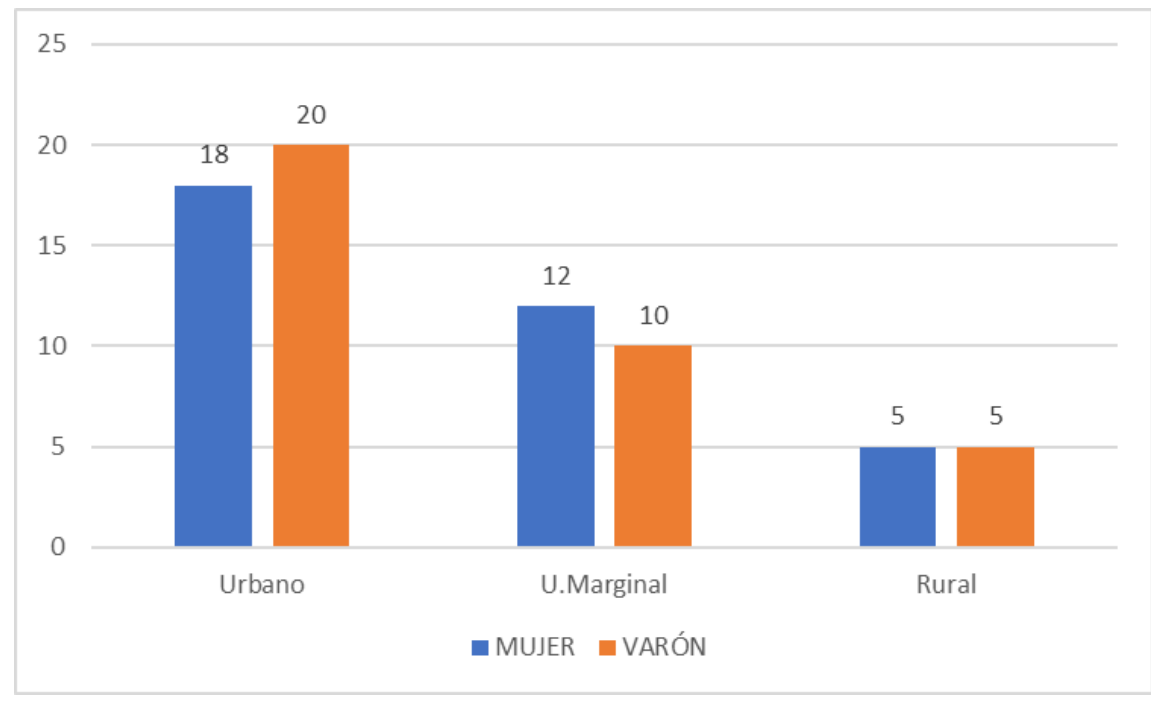

Figura 3. Variación de la actividad amilásica en pacientes altoandinos aparentemente sanos en relación a la procedencia.

El grado de amilasa en pacientes altoandinos aparentemente sanos en ambos sexos en función a la procedencia se muestra en la Figura 3. En su gran mayoría son de procedencia urbano o citadina, 18 mujeres $(25.7 \%)$ y 20 varones $(28.6 \%)$ estudian en colegios estatales y particulares de la ciudad, generalmente sus padres son empleados públicos. Ahora también hay estudiantes de la zona periférica denominada urbano marginal o barrios populares de la ciudad donde carecen de servicios básicos y son hijos de migrantes, habiendo 12 mujeres (17.2\%) y 10 varones (14.3\%). También encontramos jóvenes provenientes de otras regiones como el VRAEM que están alojados o alquilan algún cuarto, ellos vienen a estudiar para mejorar la calidad de vida de su familia, a veces quedan sin comer por los domingos por no atiende el comedor universitario donde se paga un precio mínimo o no atienden y descansan muchos restaurantes, tratan de agenciarse de 
alguna fruta o alimento, muchos son jovencitos que recién conocen la ciudad y no tienen familiares, pasan su vida sacrificando su economía.

La Tabla 4 ve reflejada los valores promedios de la actividad amilásica en pacientes altoandinos aparentemente sanos en relación al grupo etario.

\section{Tabla 4}

Valores promedios de la actividad amilásica en pacientes altoandinos aparentemente sanos en relación al grupo etario.

\begin{tabular}{lllllll}
\hline \multirow{2}{*}{ EDAD } & \multicolumn{2}{l}{ NIVELES DE AMILASA } & \multicolumn{2}{l}{ TOTALES } \\
\cline { 2 - 5 } & \multicolumn{2}{l}{ FEMENINO } & \multicolumn{2}{l}{ MASCULINO } & & \\
\hline (años) & $\mathrm{N}^{\mathrm{o}}$ & $\ddot{\mathrm{x}}$ & $\mathrm{N}^{\mathrm{o}}$ & $\ddot{\mathrm{x}}$ & $\mathrm{N}^{\mathrm{o}}$ & $\ddot{\mathrm{x}}$ \\
\hline $17-23$ & 17 & 97.9 & 11 & 95.6 & 28 & 96.8 \\
$24-30$ & 11 & 98.6 & 13 & 110.5 & 24 & 104.6 \\
$31-36$ & 7 & 146.4 & 11 & 150.6 & 18 & 148.5 \\
\hline TOTAL & 35 & 144.3 & 35 & 118.9 & 70 & 116.6 \\
\hline
\end{tabular}

Podemos precisar la variación de la actividad amilásica en pacientes altoandinos aparentemente sanos de ambos sexos según los grupos etarios en la Tabla 4. Notamos que a menos edad 17 a 23 años hay valores de $97.9 \mathrm{UA} / \mathrm{dl}$ para 17 mujeres y 95.6\% UA/dl en 11 varones. Incrementándose con la edad de 24 a 30 años en 11 mujeres (98.6 UA/dl), son superados por 13 varones (110.5 UA/dl) pero están dentro de los límites permisibles. El sector de 31 a 36 años de edad ya tiene valores superiores a los normales, por ejemplo, las 7 mujeres presentan (146.4 UA/dl) y 11 varones con $150.6 \mathrm{UA} / \mathrm{dl}$. Estos jóvenes ya casi adultos generalmente son padres jóvenes y tiene que ir a trabajar buscan un tiempo para culminar sus estudios.

De la misma manera, los valores promedios de la actividad amilásica en pacientes altoandinos aparentemente sanos en relación al índice corporal, se detalla en la Tabla 5.

Tabla 5.

Valores promedios de la actividad amilásica en pacientes altoandinos aparentemente sanos en relación al índice corporal.

\begin{tabular}{lllllll}
\hline \multirow{2}{*}{ PESO } & \multicolumn{2}{l}{ NIVELES DE AMILASA } & \multicolumn{3}{l}{ TOTALES } \\
\cline { 2 - 5 } & \multicolumn{2}{l}{ FEMENINO } & \multicolumn{2}{l}{ MASCULINO } & & \\
\hline$(\mathrm{Kg})$ & $\mathrm{N}^{\mathrm{o}}$ & $\ddot{\mathrm{x}}$ & $\mathrm{N}^{\mathrm{o}}$ & $\ddot{\mathrm{x}}$ & $\mathrm{N}^{\mathrm{o}}$ & $\ddot{\mathrm{x}}$ \\
\hline Sobrepeso & 3 & 162.0 & 10 & 160.4 & 13 & 161.2 \\
\hline
\end{tabular}




\begin{tabular}{lllllll}
\hline Normopeso & 18 & 120.6 & 14 & 177.7 & 32 & 119.2 \\
Bajo peso & 14 & 89.3 & 11 & 91.9 & 25 & 90.6 \\
\hline TOTAL & 35 & 124.0 & 35 & 123.3 & 70 & 123.7 \\
\hline
\end{tabular}

A su vez es perceptible la variación de la actividad amilásica en pacientes altoandinos aparentemente sanos de ambos sexos, según el índice de masa corporal se plasma en la Tabla 5. Los jóvenes son sobrepeso especialmente mujeres presentan valores $162 \mathrm{UA} / \mathrm{dl}$ y los varones $160.4 \mathrm{UA} / \mathrm{d}$. Estos datos son superiores a los valores normales que va a $120 \mathrm{UA} / \mathrm{dl}$, como mencionamos ellos consumen alimentos a base de carbohidratos y y bebidas alcohólicas, lo que conllevara a la postre en una pancreatitis. Los demás datos se encuentran debajo de los valores normales de las personas con normopeso y bajo peso, mujeres $89.3 \mathrm{UA} / \mathrm{dl}$ y varones con $91.9 \mathrm{UA} / \mathrm{dl}$.

\section{Referencias bibliográficas}

Armijo Zambrano, P. E., \& Atupaña Bacuy, N. R. (2018). Determinación de amilasa y lipasa como aporte para el establecimiento de valores de referencia en estudiantes de unidades educativas rurales del Cantón Riobamba. Riobamba, Ecuador: Trabajo especial de grado de la Universidad Nacional de Chimborazo para optar al título de Licenciado en Ciencias de la Salud en Laboratorio Clínico e Histopatólogico.

Basantes Procel, J. F. (2014). Determinación de glucosa, amilasa, lipasa en sangre como ayuda en el diagnóstico de pancreatitis aguda en pacientes atendidos en el Hospital Oriental, durante el período enero-junio de 2014. Riobamba, Ecuador: Trabajo especial de grado de la Universidad Nacional de Chimborazo para optar al título de Licenciatura en laboratorio clínico e histopatológico.

Garboa Pérez, L. E., \& Ochoa Díaz, L. X. (2017). Verificación del intervalo de referencia de las determinaciones de amilasa y lipasa en el laboratorio Lab-Centro Illingworth . Guayaquil, Ecuador: Trabajo especial de grado de la Universidad de Guayaquil para obtener el título de Químico Farmaceútico. 
Huaman Apaza, H. M., \& Rodríguez Huaman, H. M. (2018). Indice glicémico y carga glicémica de la papa, moraya y chuño en pobladores sanos altoandinos de la ciudad del Cusco, 2018. Cusco: Trabajo especial de grado de la Universidad Andina del Cusco para optar al título de Medico Cirujano.

Orlando, M. (2016). Evaluación de la utilidad de la amilasa y lipasa sérica en el diagnóstico y control de tratamiento de pacientes con dolor abdominal agudo de origen pancreático y no pancreático en el Northospital. Ecuador: Trabajo especial de grado de la Universidad Central del Ecuador para optar al título de Bioquímico Clínico.

Palella Stracuzzi, S., \& Martins Pestana, F. (2010). Metodología de la investigación cuantitativa. Caracas, Venezuela: FEDUPEL, Fondo Editorial de la Universidad Pedagógica Experimental Libertador.

Restrepo, C. G., \& Tascon, A. F. (2013). Estudio Cinético del enzima Alfa-Amilasa. Santiago de Cali, Colombia: Informe.

Tabernilla, D. P., Tabernilla, R. P., Guerra, O. T., \& Díaz, Y. G. (2019). Importancia del diagnóstico de la enzima alfa amilasa salival. Revista científica estudiantil 2 de Diciembre, 2(1 (2019)) , 73-81. 